\title{
Eligibility Criteria for Deep Brain Stimulation in Parkinson's Disease, Tremor, and Dystonia
}

\author{
Renato P. Munhoz, Marina Picillo, Susan H. Fox, Veronica Bruno, \\ Michel Panisset, Christopher R. Honey, Alfonso Fasano
}

\begin{abstract}
In this review, the available evidence to guide clinicians regarding eligibility for deep brain stimulation (DBS) in the main conditions in which these forms of therapy are generally indicated-Parkinson's disease (PD), tremor, and dystonia-is presented. In general, the literature shows that DBS is effective for PD, essential tremor, and idiopathic dystonia. In these cases, key points in patient selection must include the level of disability and inability to manage symptoms using the best available medical therapy. Results are, however, still not optimal when dealing with other aetiologies, such as secondary tremors and symptomatic dystonia. Also, in PD, issues such as age and neuropsychiatric profile are still debatable parameters. Overall, currently available literature is able to guide physicians on basic aspects of patient selection and indications for DBS; however, a few points are still debatable and controversial. These issues should be refined and clarified in future studies.
\end{abstract}

RÉSUMÉ: Les critères de recevabilité de la stimulation cérébrale profonde dans des cas de maladie de Parkinson, de tremblements et de dystonie. On le sait, la maladie de Parkinson (MP), des tremblements et la dystonie demeurent les principales conditions pour lesquelles la stimulation cérébrale profonde (SCP) est habituellement indiquée. Dans cette étude, nous voulons donc mettre en évidence les preuves scientifiques pouvant orienter les cliniciens dans le choix d'un traitement de SCP. En général, les ouvrages scientifiques montrent que la SCP est efficace pour traiter la MP, les tremblements essentiels et la dystonie idiopathique. Dans ces cas, le degré d'invalidité des patients ainsi que leur incapacité à contrôler ou à agir sur leurs symptômes à l'aide du meilleur traitement médical disponible constituent deux aspects clés dans un processus de sélection. Cela dit, les résultats ne sont pas encore concluants lorsqu'on traite d'autres étiologies telles que les tremblements secondaires et la dystonie symptomatique. De plus, l'âge et le profil neuropsychiatrique des patients continuent à représenter des paramètres discutables. En somme, si la littérature scientifique existante peut orienter les médecins quant aux principaux aspects présidant à la sélection des patients et fournir des précisions en matière de SCP, certains aspects demeurent néanmoins discutables et controversés. Voilà pourquoi ces derniers devraient être davantage examinés et clarifiés dans le cadre de travaux subséquents.

Keywords: deep brain stimulation, dystonia, essential tremor, movement disorders, Parkinson's disease, surgery, tremor doi:10.1017/cjn.2016.35

Can J Neurol Sci. 2016; 43: 462-471

\section{INTRODUCTION}

During the DBS Canada Day symposium held in Toronto on July 4-5, 2014, the scientific committee invited experts to share its knowledge of eligibility criteria for deep brain stimulation (DBS) for patients with the three most common current indications for these procedures: Parkinson's disease (PD), tremor, and dystonia. Experts were provided with selected topics for which they were asked to summarize the current literature and highlight what was known and what was still controversial within the field. Owing to the difficulty in extracting objective and precise evidence-based conclusions in topics with relatively limited volume of uniform outcomes data, including disorders that are sometimes rare, the information provided derives from landmark studies but also from the authors' interpretation of the best published data. Each section contains information and conclusions that may be considered debatable and controversial in some circles; however, from the authors' perspective, this is the status of the science currently.
Eventually, this particular review is meant to stimulate further reading and critical thinking, with no ambition to provide the irrefutable solutions to the proposed topics.

Each of these topics will be discussed in the following sections.

From the Morton and Gloria Shulman Movement Disorders Centre and the Edmond J. Safra Program in Parkinson's Disease (RPM, MP, SHF, VB, AF), Toronto Western Hospital, University Health Network, Toronto, Ontario, Canada; Centre for

Neurodegenerative Diseases (MP), Neuroscience Section, Department of Medicine and Surgery, University of Salerno, Salerno, Italy; André-Barbeau Movement Disorders Unit Service of Neurology (MP), Centre Hospitalier de l'Université de Montréal and Department of Medicine, Université de Montréal, Montréal, Québec, Canada; Division of

Neurosurgery (CRH), University of British Columbia, Vancouver, British Columbia, Canada.

Received August 26, 2015. Final Revisions Submitted January 25, 2016. Date of Acceptance February 15, 2016.

Correspondence to: Dr. Renato P. Munhoz, Movement Disorders Centre - Toronto Western Hospital, 399 Bathurst Street, McL7 - 419, Toronto, ON Canada M5T 2S

E-mail: renato.munhoz@uhn.ca 


\section{Eligibility Criteria fOR DBS IN PD}

\section{Background}

DBS represents a standard surgical procedure for PD patients suffering from motor fluctuations refractory to optimal medical treatment. Surgery is directed at treating motor complications that are severe enough to justify the surgical risk. The disability derived from these complications can be related to the duration and severity of either levodopa-related motor fluctuations (off periods) or dyskinesias. The Unified Parkinson's Disease Rating Scale is a validated and widely used measure of the severity of motor symptoms (part III) and motor fluctuations (part IV), used to compare pre- and postsurgical outcomes.

Three surgical targets are currently used in PD, selected depending on their suitability to the patient's profile according to demographic and clinical characteristics and impact caused by the different motor complications: subthalamic nucleus (STN), globus pallidus internus (GPi), and ventral intermediate nucleus of the thalamus (VIM).

\section{Patient Selection, Presurgical Assessment, and Safety}

Patient selection should be carried out in designated centres by a multidisciplinary team including a movement disorders specialist, neurosurgeon, neuropsychologist, psychiatrist, neuroradiologist, and nurses, all experienced in DBS. DBS candidacy is usually established according to inclusion and exclusion criteria proposed by the core assessment program for surgical interventional therapies in PD (CAPSIT-PD). ${ }^{1}$ The following factors should be carefully assessed before advocating surgery to a given patient: disease duration, age, levodopa responsiveness, type and severity of levodopa-unresponsive symptoms, cognitive and psychiatric issues, comorbid disorders, and brain magnetic resonance imaging (MRI) findings. In the present article, we review and summarize the current recommendations for each given aspect.

As recommended by the CAPSIT-PD protocol, a patient should have a disease duration of at least 5 years before being considered for surgery, allowing for atypical forms of parkinsonism to be excluded according to published consensus guidelines. $^{2-4}$ Traditionally, patients undergoing these procedures are on relatively advanced stages of the disease, with severe motor complications and a mean disease duration of 12 to 15 years. ${ }^{5}$ On the other hand, considering surgery sooner in the course of the disease would prevent or delay motor, social, and psychological disability. Recent data support considering DBS far earlier than currently applied for the early treatment of motor complications in PD (Controlled Trial of Deep Brain Stimulation in Early Patients with Parkinson's Disease study). ${ }^{6}$ However, many factors should be acknowledged in the decision of when to consider DBS, and further data are needed to establish the potential benefit of early DBS in PD. ${ }^{7}$

Although no specific age cutoff has been defined in clinical DBS studies, most studies use age as an exclusion criterion. In fact, most patients presenting the ideal profile for surgery have a relatively young onset of $\mathrm{PD}$, and are aged younger than 70 years at the time of surgery. For older cases, several considerations influencing the risk-benefit ratio should be taken into account: comorbidities, cognitive performances, prevalence of levodopa-resistant symptoms, and overall risk of surgical complications. ${ }^{5,8}$ Although recent evidence suggests that DBS is safe in the short-term follow-up of PD patients older than age 75, data regarding long-term outcome are lacking. ${ }^{9}$ Finally, more conservative surgical approaches may be considered for elderly PD patients (unilateral vs bilateral procedures and the choice of target, GPi been considered safer than STN). ${ }^{5,8}$

Preoperative levodopa responsiveness is reported as the best predictive factor for a positive response to surgery. ${ }^{8}$ Thus, it is mandatory to perform a levodopa challenge in each surgical candidate to verify and explain to the patient what signs and symptoms are potentially responsive to surgery. A sufficient dose of levodopa to reproduce the patient's best on response, given after a medication-free interval of 12 hours, should be used to determine responsiveness. A single supra-threshold dose of levodopa is often used, typically 1.5 times the early morning dose. $^{5}$ According to the CAPSIT-PD, the test should induce at least a 33\% decrease in the Unified Parkinson's Disease Rating Scale, part III, total score. ${ }^{1}$

Levodopa-resistant features, including gait and balance issues, dysarthria, and dysphagia, usually do not improve or may even worsen after DBS. Type and severity of these symptoms should be assessed during the levodopa challenge at a time when the patient and examiner agree that the peak of optimal benefit has been obtained. In these patients, levodopa-resistant features might jeopardize the outcome of DBS. However, surgery might still be an option, but only after careful weighing of expected benefits, residual disability, and risks. ${ }^{5,8}$ Severe tremor is an exemption because it can be poorly responsive to levodopa but improves with both STN and VIM DBS surgery. ${ }^{8}$

Cognitive dysfunction and psychiatric issues affect almost all PD patients and tend to be more severe as the disease progresses. It is critical to establish the extent of cognitive impairment and behavioural issues that might not allow a safe surgery outcome. ${ }^{10}$ Thus, a thorough preoperative assessment is mandatory, although there is insufficient evidence to make definitive conclusions about the predictive validity of any given assessment, interview, or test. ${ }^{5,10}$ Regarding cognition, dementia is an absolute contraindication for surgery. ${ }^{5}$ On the other hand, there are no clear recommendations regarding mild cognitive impairment. With respect to psychiatric disturbances, surgery is generally deferred in patients with unstable psychiatric conditions until the symptoms have been managed adequately. This is particularly true for depression and psychosis. Ongoing severe depression with suicidal ideation should be considered an absolute contraindication to surgery because of an increased risk of suicide in the first year after the procedure. Limited evidence suggests that GPi may be safer than STN for patients with either mild cognitive or behavioural issues. ${ }^{10,11}$

Patients with unstable severe health issues are commonly not considered surgical candidates; thus, there are no data regarding this subset of patients. However, although formal studies are lacking, serious comorbidities should be regarded as a contraindication to DBS given the negative influence on the risk-benefit ratio. ${ }^{5}$

In patients being considered for surgery, preoperative imaging, usually MRI, is mandatory. Although some centres choose to avoid surgery in patients with preoperative findings of extensive atrophy and cerebral vasculopathy, conclusive data are lacking. ${ }^{5}$ Of note, severe cortical atrophy increases the risk of postoperative subdural hematomas. ${ }^{8}$ However, aside from obvious structural lesions, imaging findings alone should not be considered absolute contraindications to DBS. ${ }^{5}$ 
Finally, a careful evaluation of personal, professional, and social issues of each patient is fundamental for achieving a good outcome after DBS. The periods prior, during, and after the surgery require a great amount of cooperation and motivation from patients and caregivers. The absence of a solid support from a caregiver should be considered a reason to preclude DBS.

In conclusion, several factors should be taken into account when considering surgery in PD patients and a thorough evaluation of the risk-benefit by a specialized multidisciplinary team is mandatory in all cases.

\section{Eligibility Criteria For DBS in Dystonia}

\section{Background}

The term "dystonia" encompasses a heterogeneous group of movement disorders characterized by sustained or intermittent muscle contractions causing twisting and repetitive movement, abnormal postures, or both. A recent revision now classifies dystonia into two axes: (1) clinical characteristics (age at onset, temporal pattern, body distribution, whether focal, segmental, or generalised; and associated features) and (2) aetiology, whether idiopathic/genetic or secondary to other neurological/medical diseases. $^{12}$

Pharmacological treatments for dystonia remain generally unsatisfactory. The treatment of generalized dystonia is usually based on the use of various combinations of levodopa, anticholinergics, and muscle-relaxing drugs. Overall outcomes are poor because of a lack of efficacy and side effects. For focal or segmental dystonia, the preferred treatment is the local injection of botulinum toxin to denervate the affected muscles. ${ }^{13}$ Bilateral GPi DBS surgery has shown to be a useful strategy in both generalized and focal dystonia. ${ }^{14} \mathrm{~A}$ rigorous selection of the patients and a strict control of comorbidities are essential to predict a good outcome. The level of evidence for this treatment has grown in recent years. ${ }^{15,16}$

\section{Indications and Presurgical Assessment}

Criteria for selecting patients for GPi DBS for dystonia remain ambiguous. In general, surgical referral for all types of dystonia can be considered in patients who have failed trials with anticholinergic drugs, benzodiazepines, and levodopa in generalized/segmental dystonia, or had no benefit or failure with botulinum toxin injections in cranial and cervical dystonia. There is currently no widely accepted consensus about which type of medication, which dose, or how many trials are needed before surgery. In general, it is not mandatory to have tried all available medications.

Symptoms should be disabling enough to justify the surgical risk. The Burke-Fahn-Marsden (BFMD) Dystonia Rating Scale ${ }^{17}$ and the Toronto Western Spasmodic Torticollis Rating Scale (TWSTRS) $^{18}$ are two validated and widely used scales used to measure dystonia disability and compare pre- and postoperatory outcomes. However, there is no agreement about which scales to use to assess symptoms, or which threshold scores for disability, dystonia, and pain severity are needed for surgery. During the preoperatory assessment, it is generally important to consider using quality of life $(\mathrm{QoL})$ scales, as this is often the main reason for surgery.
Preoperatory investigations usually involve imaging, although there is no special technique requirement. Usually, a brain MRI scan is required to support the diagnosis of idiopathic or secondary dystonia. The presence of minor structural abnormalities in the basal ganglia in idiopathic dystonia is not a contraindication for DBS. In cervical dystonia, recurrent involuntary neck movements can often exacerbate osteoarthritic cervical spondylosis, which may be an independent factor causing neck pain. To assess the degree of arthritic spinal degeneration and any need for spinal surgery before or after DBS, a cervical spine MRI scan may be needed. In addition, skeletal imaging might be useful to quantify spinal deformities that are common in children with dystonia secondary to GAG deletion in the TOR1A gene (DYT-1)-related disease. ${ }^{19}$

Other evaluations that should be considered include a complete neuropsychology/psychiatric assessment before and after surgery. This is especially important in selected patients with psychiatric comorbidities because of high psychiatric comorbidity in the dystonia population and a few suicides have been reported after GPi DBS. ${ }^{20}$

\section{Current Evidence and Controversial Issues \\ Idiopathic Dystonia}

Currently, there is level I evidence for benefit of DBS in inherited/idiopathic generalized/segmental dystonia. This level of evidence was reached in one class I study and one class II study showing $40 \%$ to $50 \%$ improved after 3 months in shamcontrolled, double-blind evaluations. ${ }^{21,22}$ In 2005, Vidailhet et al reported 22 patients with idiopathic generalized dystonia who had a significant improvement in dystonia severity with GPi DBS in a double-blind evaluation with and without stimulation at 3 months of follow-up in a multicentre, prospective, controlled study. ${ }^{21}$ A year later, Kupsch et al published a multicenter, randomized, sham-controlled study that showed a significant improvement in the severity of dystonia in 20 patients who had generalized/ segmental idiopathic dystonia at 3-month follow-up after bilateral GPiDBS. $^{22}$ In 2012, Volkmann et al showed that 3 years and 5 years after surgery, pallidal stimulation continued to be an effective and relatively safe treatment option for patients with severe idiopathic dystonia, with a $50 \%$ to $60 \%$ improvement. $^{23}$ In this group of patients, the accumulated experience of these studies suggests that younger age at time of surgery, and less severe dystonia are correlated with better motor improvement but not with function. In addition, postoperative pallidotomy-like effects, mobile versus fixed, and the presence of contractures are not predictive of outcome. ${ }^{24}$

DYT-1-positive dystonia is typically regarded as responding better to pallidal procedures. This observation derives from several studies that showed earlier and greater improvements in DYT-1-positive dystonia patients when compared with non-DYT-1 related cases. ${ }^{21,22}$ More recent studies, however, demonstrated that DYT-1-positive cases are operated on at a younger age and with shorter disease duration, which are both well-recognized predictors of good outcome after stereotactic surgery for dystonia. Therefore, the impact of genetic status per se may not be as strong as the inherent biases found in these cases. ${ }^{25}$

In cervical dystonia, a single class I randomised, sham-controlled trial by Volkmann et al showed that after 3 months of bilateral GPi DBS, CD severity and related disability were reduced by $26 \%{ }^{26}$ In addition, there are four published 
class III studies assessing a total of 32 patients, with a follow-up between 12 and 60 months. On average, these four studies showed $50 \%$ improvement in the TWSTRS. ${ }^{27-30}$

\section{Safety}

The previously described studies reported that GPi DBS is a generally well-tolerated procedure. However, speech difficulties deserve attention, especially in patients with previous speech disturbances, because dysarthria has been found in most of the reported series. Permanent speech problems were reported in four patients in retrospective series and the number increased to seven of 60 patients in the Volkmann et al prospective study. ${ }^{15,26}$ Other complications included transient lethargy/somnolence/stupor, transient involuntary movements disorders, transient hemiparesis, cervical pain, electrode dislocation and misplacement, and a depressive episode requiring psychiatric treatment. ${ }^{15,21-30}$

\section{Secondary Dystonia}

In general, there is less evidence for the effectiveness of DBS in secondary and neurodegenerative dystonias than for idiopathic focal generalized/segmental forms. This is for the most part from initial improvement, which is often followed by gradual and consistent worsening related to progression of the ongoing underlying disease. Individuals with neurodegenerative dystonias (resulting from brain pathology) are also thought to be at greater risk of side effects from DBS. In particular, there are currently no specific and clear guidelines defining suitable patients with secondary dystonia that should be referred for surgery.

Tardive dystonia can cause significant disability in some individuals and surgical treatment has been performed with some success. In 2007, Damier et al published a class III study demonstrating effectiveness of bilateral GPi DBS in this type of dystonia. The results were confirmed by a double-blind evaluation of 10 patients showing a mean decrease of 50\% in the Extrapyramidal Symptoms Rating Scale score when stimulation was applied compared with the absence of stimulation in a 6-month time frame. ${ }^{31}$ Lately a systematic review including 17 articles with 50 patients confirmed the efficacy and safety of this treatment to tardive dystonia. ${ }^{32}$

In cerebral palsy $(\mathrm{CP})$, there is some evidence for mild improvement. In a multicentre prospective pilot study, bilateral GPi DBS was performed in 13 adults with dystoniachoreoathetosis CP. Results showed an improvement in the mean BFMD rating scale movement score from a mean 44.2 before surgery to 34.7 at 1 year postoperatively. Functional disability, pain, and mental health-related QoL were significantly improved. It is important to highlight that these patients did not experience any worsening of cognition or mood. ${ }^{33}$ A meta-analysis of 20 articles and a total of 68 patients with $\mathrm{CP}$ treated with GPi DBS found improvements in the BFMD motor scale of $23.6 \%$ after a mean follow-up of 12 months; the improvement in the disability component of the same scale was less impressive but still significant, 9.2\%. The authors also found a significant negative correlation between severity of dystonia and clinical outcome. $^{34}$

Finally, there was one study (class III) for DBS in eight patients with secondary dystonia with different aetiologies (CP, postencephalitis, postanoxic damage, sepsis, neuroleptics, stroke, radiation treatment). ${ }^{35}$ Results were overall positive, showing improvement in six subjects on the Unified Dystonia Rating Scale (the mean preoperative score was 40 ; postoperative score was 26.4); however, this study confirmed clinical experience that secondary/neurodegenerative dystonias generally respond less well than idiopathic dystonia to GPi DBS, with the exception of tardive dystonia. ${ }^{35}$

\section{Safety}

In reported series from patients with secondary dystonia of various etiologies, adverse effects of the GPi DBS appear to be similar to those with idiopathic dystonia. Most were related to the surgical procedure. Of 50 patients who received GPi DBS for tardive dystonia, one experienced an exacerbation of depression and another experienced an exacerbation of psychosis. ${ }^{32}$ From the series of patients with $\mathrm{CP}$, one patient experienced transient postoperative bronchospasm and confusion that resolved during the hospital stay of 7 days and one experienced worsening of dystonia. ${ }^{28,33}$

\section{Conclusions}

In summary, GPi DBS is now well-established for the treatment of generalized or segmental idiopathic dystonia after failure of medical treatment, although the definition of "failure" has not yet been determined. Patients with idiopathic/inherited dystonias are likely to have the best outcome from DBS surgery, especially those who are younger, DYT-1 positive, with a shorter disease duration, and have less severe dystonia. Likewise, in focal cervical dystonia after failure of botulinum toxin injection, DBS can be considered a good option. For patients with secondary dystonias, the surgical alternative should be carefully considered, because it is generally less effective, although tardive dystonia does appear to respond better. A specialized surgical team assessment is required in all cases.

\section{Eligibility Criteria for Deep Brain Stimulation in TrEMOR}

\section{Background}

Tremor is an involuntary movement characterized by a rhythmic oscillation about a fixed point or trajectory. It can be classified based on its clinical features or underlying cause. ${ }^{36}$ Treatment is symptomatic and tremor has been the first approved indication for DBS, with clinical experience accumulating now for almost three decades. ${ }^{37}$ Because bilateral lesions of the thalamus are very often associated with irreversible side effects, VIM of the thalamus DBS was initially proposed in the 1980s for patients with unilateral thalamotomy requiring bilateral procedures. ${ }^{38}$ Given the tradition of thalamotomies and intraoperative recordings (tremor cells), the thalamus is virtually the target of choice for most tremor conditions. ${ }^{39}$ The standard stereotactic coordinates for thalamic DBS cover a region in of the motor thalamus known as VIM nucleus (Fig. 1). Unilateral procedures (targeting the thalamus contralateral to the dominant and/or most affected hand) are usually performed. However, bilateral procedures may be considered especially when head, voice, or trunk tremors are the main indications for surgery. Following successful DBS for control of tremor in the dominant hand, some patients decide they do not require the other side to be done. As a result, some centres have adopted an approach that entails staging the procedures. This 


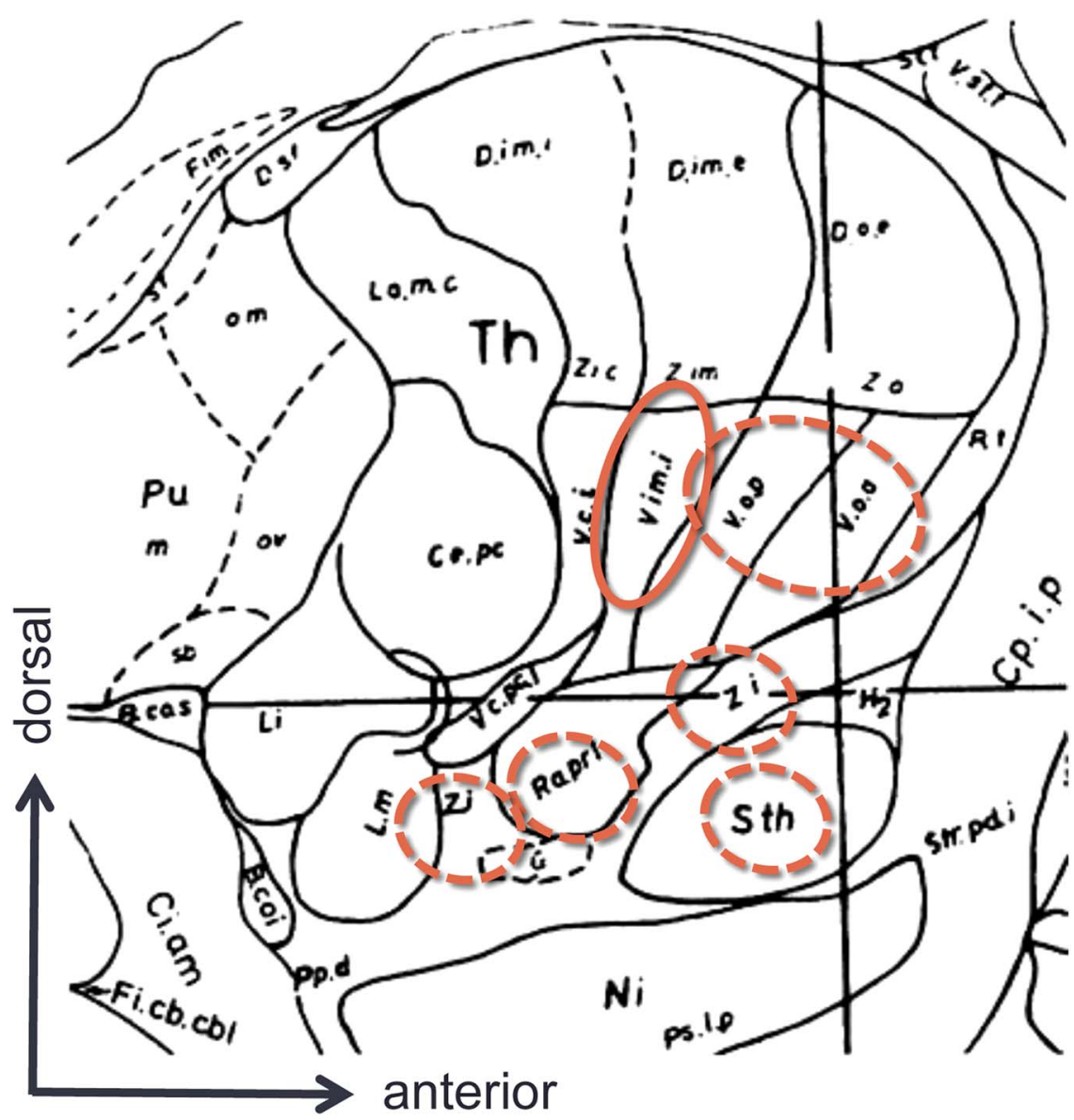

Figure 1: Targets for surgical treatment of tremor. A sagittal slice from the SchaltenbrandWahren Atlas. The standard target is the Vim; experimental/novel targets are shown by the dotted lines. Cp.i.p, capsula interna crus posterior; $\mathrm{H}_{2}$, campus foreli pars $\mathrm{H}_{2}$; $\mathrm{Ni}$, nucleus niger; Ra.prl, radiation prelemniscalis; Sth, corpus subthalamicum; V.c.i, nucleus ventrocaudalis internus; Vim.i, nucleus ventrointermedius internus; V.oa, nucleus ventro-oralis anterior; V.op, nucleus ventro-oralis posterior; Zi, zona incerta.

approach may help to identify some patients who benefit enough from a unilateral procedure not to require contralateral surgery. Furthermore, the time interval between the two stages favors functional compensation and recovery, and diminishes the negative consequences associated with a second procedure. ${ }^{39}$

\section{Selection Criteria: Clinical Evidence}

The decision as to whether a patient with tremor is appropriate for DBS is complex and multifaceted. ${ }^{39,40}$ The first question is whether there is a chance for successful medical treatment. If the established medications at the highest tolerated dose have been tried (e.g. propranolol and primidone for ET, levodopa for PD, botulinum toxin injections for dystonic tremor) without success, then surgery might be considered. Two main factors have to be taken into account at this point: the specific tremor condition and patient's features ${ }^{39}$; therefore, reaching the right diagnosis is one of the most important factors in this process because many of those seeking surgery due to the lack of benefit from medication are not in fact affected by tremor (e.g. myoclonus in cortical tremor or limb ataxia in cerebellar diseases) or, more importantly, have a functional tremor.

\section{$E T$}

ET is the most frequent movement disorder, and the majority of patients suffer from tremor of the hands and arms; approximately $40 \%$ suffer from head and $20 \%$ from voice tremor. ${ }^{36}$ The majority of candidates for functional neurosurgery belong to the ET subgroup suffering from severe intention tremor. In the vast majority, unilateral VIM DBS may be sufficient to reduce disability by suppressing tremor within the dominant hand. ${ }^{37}$ However, in cases with disabling bilateral limb tremor or head, voice, and trunk tremor, a bilateral procedure may be indicated. VIM DBS is highly effective and, as a rule of thumb, contralateral tremor scores decrease by $80 \%$ in $80 \%$ of patients; this is also confirmed by long-term studies (up to 8 years after surgery); zonaincerta, see Deuschl et $\mathrm{al}^{37}$ ).

\section{Parkinsonian Tremor}

PD patients might display severe resting tremor (PD tremor type I) resistant to high doses of levodopa or disabling ET-like action tremor (PD tremor type II) that does not respond to dopaminergic drugs. 
DBS studies have shown that tremor-dominant $\mathrm{PD}$ is successfully relieved by stimulation of the VIM or STN. VIM DBS has been shown to improve resting and action tremors but will not affect other cardinal features of PD, whereas STN DBS has been shown to improve all three cardinal signs of PD. ${ }^{41} \mathrm{GPi}$ DBS also has antitremor effects, as well as improving dyskinesia, akinesia, and rigidity. ${ }^{41}$ Because of the lack of benefit for bradykinesia and rigidity, VIM DBS is not recommended routinely but can be considered for tremor-dominant elderly patients with slow disease progression in whom other features of PD are not a source of disability. In fact, VIM DBS is safer than STN DBS and can be performed unilaterally. In addition, postoperatively, many patients can significantly reduce the high doses of dopaminergic medications used to control the tremor, thus avoiding their side effects (e.g. hallucinations, confusion), which are particularly common in the elderly. It has been suggested that VIM DBS could be a therapeutic option particularly for these patients. ${ }^{39}$

\section{Dystonic Tremor}

There is minimal available information about the treatment of the dystonic tremor syndromes and tremor associated with dystonia in particular. In fact, reports on the treatment of dystonic tremors are rare because the target symptom in these cases is usually dystonia; tremor is not even specifically itemized in the most commonly deployed dystonia rating scales. ${ }^{42}$ Dystonic tremor can improve after GPi or VIM DBS (for a review on the outcome see Fasano et $\mathrm{al}^{42}$ ). Primary writing tremor might be considered as a task-specific focal dystonia and it has been reported to be successfully treated with VIM DBS. ${ }^{42}$

\section{Holmes Tremor}

Holmes tremor is characterized by a low-frequency rest tremor $(<4.5 \mathrm{~Hz})$ accentuated by posture and intentional movements, usually arising 4 weeks to 2 years after an acute brain event. Lesions typically involve the cerebello-thalamo-cortical and dentato-rubro-olivary pathways with additional dysfunction in the nigrostriatal pathway, accounting for the rest tremor component. Causes include hemorrhage, tumors, cavernomas, infection, multiple sclerosis, or trauma. The effects of medical treatment are highly variable and include levodopa, dopamine agonists, or anticholinergics, which may be useful when the parkinsonian tremor prevails; however, it is well known that medical treatment of Holmes tremor is most times unsuccessful. Few cases of Holmes tremor have had VIM DBS with a generally good response but, as with cerebellar tremor, much depends on the extent of the natural lesion and the specific symptom patterns in the individual patient.

A recent report of ten patients with refractory Holmes tremor who underwent either VIM or GPi DBS showed significant improvements for both targets of stimulation with an average improvement of 64\%, sustained at 2 years' follow-up. Of importance, the choice of target was based results of intraoperative stimulation. $^{43}$

\section{Multiple Sclerosis-Associated Tremor}

In patients with multiple sclerosis (MS), upper limb intention tremor is less prevalent than postural tremor, but is regarded as the predominant source of disability and an important factor in the context of assessment for functional neurosurgery. ${ }^{40,44}$ Intention tremor is the consequence of a cerebellar dysfunction and, as such, is difficult to separate from other features such as proximal postural instability or dysmetria. Another symptom of cerebellar tremor is titubation, which presents as a low-frequency oscillation of the head or trunk that in general responds poorly to DBS. Selecting patients with tremor associated with MS is a very difficult task. ${ }^{43,44}$ Once again, tremor is considered to improve with DBS, whereas ataxia does not. This distinction can be very difficult to make particularly for movements with frequencies below $3 \mathrm{~Hz}$. In practice, the degree of rhythmicity of the movement disorder can be a useful distinguishing criterion. ${ }^{46}$

The results of VIM DBS for MS tremor might be encouraging taking into account the quantitative measures of tremor; however, disability and quality of life are only rarely improved. ${ }^{45}$ In a subgroup of carefully selected patients (who have no superimposed ataxia, weakness, or sensory loss in the tremulous limb, with shorter disease duration and less severe and purely distal preoperative tremor), thalamic DBS may result in a reasonable reduction of postural and intention tremor.

\section{Other Cerebellar Tremors}

Cerebellar tremor may be the consequence of head trauma, stroke, or degenerative diseases. The phenomenology is variable and resembles MS tremor and, again, medical treatment is usually unrewarding. Cerebellar tremors, of various etiologies, are classically treated with VIM DBS and in a few patients, substantial improvement has been reported.

A particular type of cerebellar tremor (in variable combination with ET-like and/or parkinsonian tremor) is seen in patients carrying the premutation for Fragile $\mathrm{X}$ syndrome (Fragile $\mathrm{X}$-associated tremor/ataxia syndrome). Few patients have benefited from unilateral VIM DBS, whereas bilateral procedures seem to be associated with worsening of ataxia. ${ }^{47,48}$

\section{Tremor in Peripheral Neuropathy}

Many chronic peripheral, often demyelinating, neuropathies are accompanied by tremors, which are usually mild postural tremors, although some cases suffer from very disabling action and intention tremors. Medical treatment is primarily concerned with treating the underlying peripheral neuropathy. However, even when the neuropathy responds, the tremor may not or may even worsen. Few patients with paraproteinaemic demyelinating neuropathy have been reported to be successful treated with unilateral VIM DBS. ${ }^{49,50}$

\section{Orthostatic Tremor}

From the few patients described to date, this tremor seems to respond to bilateral VIM DBS; in fact, patients with unilateral procedure gained benefit only after the other hemisphere had been implanted. ${ }^{51,52}$ Interestingly, although disability and patient's perception respond to DBS, less evident is the effect of surgery on the actual amplitude of the tremor.

\section{Palatal Tremor}

This tremor can be extremely disabling, especially in secondary forms that can be associated with other cranial tremors that can 
cause oscillopsia in case of eye involvement. So far, only one case has been described and no benefit was obtained with DBS of red nucleus. $^{53}$

\section{Patient-Related Factors}

Age

Reported case-series of VIM DBS often include subjects up to 80 years of age or more. However, we consider the ideal candidate for DBS to be "biologically" younger than age 75 years, despite the fact that there is insufficient evidence from published data to indicate that the results of thalamic DBS are affected by this variable. $^{39}$

\section{Disability and Functional Gain}

Patient selection is individualized depending on the status of the patient, employability, interpersonal relationships, and expectations of functional gain. In fact, the most important outcome for the patient is an improved function. In terms of pragmatic assessment, disability becomes unacceptable if tremor significantly interferes with feeding (thus causing weight loss), drinking, and writing, or, in the case of vocal or head tremor, disrupts communication. On the other hand, disability sometimes derives exclusively from the social impact of a tremor, for instance when it is socially stigmatizing.

\section{Patient's Perspectives and Expectations}

Patients are often intolerant of antitremor medication, even at low or standard doses, or simply do not want to take medications. Each patient has to balance the potential benefit against the potential risks of DBS. In fact, even in the best scenario, DBS is not risk-free. The approximate risk of an intracranial hemorrhage causing a permanent neurological deficit is below $1 \%$ and the general risk of a surgery-related death is considered to be between $1: 1000$ and $1: 10,000$. In addition, there are also minor risks to consider, namely system failure, infection, and seizures after surgery. Moreover, patients need to understand that regular follow-up and minor surgical procedures (replacement of the impulse generator when the battery expires) are required after surgery. Another fundamental issue is patient's expectation, which has to be realistic. Patients have to know that VIM DBS may help contralateral distal tremor, whereas the effect on proximal tremors is less reliable. In most cases, head and voice tremor might benefit only after bilateral implants. However, gait ataxia, dysarthria, and cognition may worsen after bilateral procedures.

\section{Systemic Comorbidities}

Although formal studies are lacking, serious systemic comorbidities should be regarded as contraindications to DBS. These include all disease that markedly reduces life expectancy or would significantly compromise the benefits of DBS or amplify the risks of surgery.

\section{Neurological Comorbidities}

Dementia, major psychotic disorders, severe personality disorders, and alcoholism are usually considered contraindications to DBS. Major depression and anxiety disorders should have been effectively treated before the procedure is undertaken. In nondemented patients, thalamic DBS is considered a relatively safe procedure because only minor deteriorations in verbal memory and fluency have been described and there is no evidence of other negative neuropsychological consequences. Because dysarthria and dysphagia are common side effects of thalamic DBS, caution should be exercised when considering patients who have these symptoms preoperatively.

MS patients represent a delicate condition because they suffer from a dynamic condition. MS exacerbations have been reported after DBS implantation, but the available data do not clearly indicate whether they represent a change in exacerbation frequency relative to presurgical levels. For this reason, patients with MS should only come to surgery if the disease has been clinically stable for about 12 months. ${ }^{39}$

\section{Previous Surgery for Tremor}

There is a paucity of data available about DBS in patients who have had a previous surgical procedure for tremor. However, successful unilateral and bilateral DBS have been reported in patients with previous unilateral pallidotomy or thalamotomy, although adverse effects are frequently reported particularly when a previous contralateral lesion is present.

\section{Areas of Controversy and Future Directions}

In spite of the thousands of patients implanted worldwide and a fair amount of clinical studies, many areas of controversy are still present. Future researches and randomized clinical trials (RCT) should work on these still unsolved issues.

\section{When to Define a Medication-Refractory Tremor?}

There are no established criteria for the drugs to be used before considering surgery. One approach is to test all the drugs that have been proven to be effective in RCTs for a particular tremor in each patient, in a strict evidence-based approach. ${ }^{39}$ As for ET, its present medical treatment involves numerous drugs, although only some have been properly studied. According to American Academy of Neurology guidelines, primidone and propranolol should be offered to patients who desire treatment for limb tremor in ET with level A evidence. ${ }^{54}$ The maximum tolerated dose and even their combination should be tried before considering surgery in ET.

As for PD, a levodopa challenge is useful in confirming the diagnosis, but not for the definition of medication-refractory tremor because parkinsonian tremor is highly fluctuating and might only respond to very high doses. Clozapine might be considered in such cases, at least before proposing DBS. ${ }^{55}$

As for dystonic tremor, drugs are generally disappointing, but botulinum toxin injections might be particularly helpful-and should be tried-when it comes to head or voice tremor. ${ }^{42}$

As for other tremors, RCTs are generally not available. Consequently, the use of medical treatments is usually based on small open-label studies or case reports. In practice, we use the following algorithm before considering a patient refractory to medical therapy: propranolol, primidone, propranolol and primidone together, topiramate, a single dose of clozapine for hand and head tremor, and botulinum toxin injections for head and voice tremor. ${ }^{39,40}$ 


\section{What Is the Evidence Supporting Using VIM DBS?}

Most evidence has been built for ET and PD and in old RCTs with a relatively short follow-up. Therefore, in spite of an effect superior to aforementioned drugs, the American Academy of Neurology level of recommendation for VIM DBS goes from $\mathrm{C}$ to $\mathrm{U}$ (insufficient evidence) and only considers ET and unilateral procedures. Even the supposed superiority on thalamotomy has been questioned in recent years. ${ }^{54}$ An important weakness of DBS is the phenomenon of benefit decay over years, which occurs relatively often and should be declared to patients considering surgery. ${ }^{56}$ The pathophysiology of such decay is still unclear, and two main hypotheses have been considered: habituation to DBS or disease progression. ${ }^{57}$

\section{Target(s) of Choice}

Historically, the first-choice target for alleviating tremor was the VIM nucleus. It should be considered in almost all types of tremor with two important exceptions: dystonic tremor in the context of disabling dystonic postures and parkinsonian tremor in the context of disabling bradykinesia and/or rigidity. In these diseases, the suggested targets are GPi and STN, respectively. An important question is whether either dystonia or parkinsonism will progress beyond tremor. For instance, a specific subgroup of PD patients with "monosymptomatic rest tremor" present with a classical rest tremor but without sufficient evidence of akinesia, rigidity, or postural disturbance to confirm a diagnosis of PD. Long-term observations of cohorts of these patients have shown that they may develop the full-blown picture of PD after a period of 10 years or more. It is debatable whether these patients should undergo VIM or STN DBS.

Recent evidences point to the ventral border of the VIM and the adjacent subthalamic white matter (Zona incerta, prelemniscal radiation) as the best target for ET, because this is an anatomical bottleneck where stimulation engages most fibers directed to the motor thalamus (Fig. 1). ${ }^{58}$ This would turn into a more favorable outcome for proximal tremor and concomitant ataxic features. A few studies have pointed out that a more anterior location of the stimulation electrode within ventralis oralis posterior, receiving afferents from GPi, may also be effective especially for attenuating intention tremor (Fig. 1). Tremor in the proximal arm muscles, trunk, or legs may not respond as well as distal arm tremor to thalamic DBS; there are some small case series reporting that stimulation within the posterior subthalamic area (caudal zona incerta) may also alleviate this kind of tremor. There is also a report of DBS of the subthalamic white matter remarkably improving proximal dystonic tremor that had been refractory to Vim thalamotomy. Very limited is the knowledge of other targets, such as ventralis oralis anterior or STN in patients without PD. In one case, bilateral STN DBS has been reported to improve cervical dystonia, dystonic head tremor, and ET-like tremor of the hands.

Targeting less standard zones might be relevant especially for patients with a cerebellar tremor. VIM-DBS has been considered the target of choice for Holmes tremor. However, based on the hypothesis that Holmes tremor is caused by the combined imbalance of different cerebral circuits, different double approaches have been used in single cases: VIM DBS variably combined with STN, GPi, ventralis oralis posterior, and/or ventralis oralis anterior. ${ }^{58}$ Likewise, some studies suggest that stimulation within the subthalamic white matter might be superior to stimulation of the thalamus in MS-related tremor.

\section{What Is the Role of Novel Surgical Approaches?}

Besides standard thalamotomy, noninvasive thalamotomies have been developed in recent years. After the initial enthusiasm, gamma knife thalamotomy is less often taken into consideration because of the use of radiation and the unpredictability of efficacy and side effects. ${ }^{59} \mathrm{~A}$ new and interesting approach is focused magnetic resonance-guided ultrasound thalamotomy, which has immediate effects and does not use radiation. ${ }^{60}$ The pros and cons of such approaches with respect to VIM DBS are still unknown and presently this treatment is considered experimental and mainly offered to patients who cannot undergo DBS and/or do not want invasive treatments.

\section{Can Preoperative Testing Predict DBS Outcome?}

Response to VIM DBS is extremely variable especially for patients without ET or PD. Tremor patients who are under consideration for DBS should be evaluated using a multidisciplinary team approach. It has been suggested that preoperative physiological techniques (e.g. use of accelerometer) are able to discern which patients with MS are likely to benefit from DBS. Distal tremors, with a frequency above $3 \mathrm{~Hz}$, appearing as a single peak in the frequency spectra, may be more effectively suppressed. ${ }^{44,45}$ As for preoperative neuroimaging, besides the mandatory brain MRI, other imaging studies may be useful. 18-F-dopa positron emission tomography or dopamine transporter single-photon emission computed tomography might be considered to: (1) confirm a clinical diagnosis of ET because STN rather than the VIM is usually the preferred target for patients with an underlying dopaminergic deficit; (2) discriminate dystonic tremor from "monosymptomatic rest tremor" from underlying PD; and (3) confirm a clinical diagnosis of Holmes tremor. Finally, it is still impossible to predict long-term benefit decay, and future studies should address this issue as well.

\section{DisCLOSURES}

MP has received a grant from the Michael J. Fox Foundation for Parkinson's Research, and is an employee of and received a salary from the University Health Network, Toronto, Canada (UHN) and the University of Salerno. $\mathrm{CH}$ is a speaker and consultant for and has received honoraria and consulting fees from Medtronic. AF is a consultant and advisor for and receives consulting fees and honoraria from Abbvie, Boston Scientific, TEVA Canada, Novartis, UCB Pharma, and Medtronic; he also is a grant recipient and receives research support and honoraria from Medtronic. The other authors have nothing to disclose.

\section{ACKNOWLEDGEMENTS}

This review is the product of the discussion between Canadian deep brain stimulation experts occurred during the DBS Canada Days held in Toronto on July 4-5, 2014. Dr. Mark Guttman from the Centre for Movement Disorders, Markham, Ontario, collaborated with the discussion of these topics during the above referred meeting.

Although the symposium was sponsored by Medtronic and all speakers received honoraria and necessary transportation 
and accommodation, the final report provided has no industry-specific recommendations and was screened to avoid potential bias.

\section{REFERENCES}

1. Defer GL, Widner H, Marie RM, Remy $P$, Levivier $M$. Core assessment program for surgical interventional therapies in Parkinson's disease (CAPSIT-PD). Mov Disord. 1999;14: $572-84$

2. Litvan I, Agid Y, Calne D, et al. Clinical research criteria for the diagnosis of progressive supranuclear palsy (Steele-RichardsonOlszewski syndrome). Report of the NINDS-SPSP InternationalWorkshop. Neurology. 1996;47:1-9.

3. Gilman S, Wenning GK, Low PA, et al. Second consensus statement on the diagnosis of multiple system atrophy. Neurology. 2008;71:670-6.

4. McKeith IG, Dickson DW, Lowe J, et al. Diagnosis and management of dementia with Lewy bodies: third report of the DLB Consortium. Neurology. 2005;65:1863-72.

5. Pollak P. Deep brain stimulation for Parkinson's disease - patient selection. Handb Clin Neurol. 2013;116:97-105.

6. Schuepbach WM, Rau J, Knudsen K, et al. Neurostimulation for Parkinson's disease with early motor complications. N Engl J Med. 2013;368:610-22.

7. Mestre TA, Espay AJ, Marras C, Eckman MH, Pollak P, Lang AE. Subthalamic nucleus-deep brain stimulation for early motor complications in Parkinson's disease-the EARLYSTIM trial: early is not always better. Mov Disord. 2014;29:1751-6.

8. Munhoz RP, Cerasa A, Okun MS. Surgical treatment of dyskinesia in Parkinson's disease. Front Neurol. 2014;5:65.

9. DeLong MR, Huang KT, Gallis J, et al. effect of advancing age on outcomes of deep brain stimulation for Parkinson eisease. JAMA Neurol. 2014;71:1290-5.

10. Voon V, Kubu C, Krack P, Houeto JL, Tröster AI. Deep brain stimulation: neuropsychological and neuropsychiatric issues. Mov Disord. 2006;21(Suppl 14):S305-27.

11. Castrioto A, Lhommée E, Moro E, Krack P. Mood and behavioral effects of subthalamic stimulation in Parkinson's disease. Lancet Neurol. 2014;13:287-305.

12. Albanese A, Bhatia K, Bressman SB, et al. Phenomenology and classification of dystonia: a consensus update. Mov Disord. 2013;28:863-73.

13. Albanese A, Asmus F, Bhatia KP, et al. EFNS guidelines on diagnosis and treatment of primary dystonias. Eur $\mathrm{J}$ Neurol. 2011;18:5-18

14. National Institute for Health and Clinical Excellence (NICE). Deep brain stimulation for tremor and dystonia (excluding Parkinson's disease). http://www.nice.org.uk/Guidance/ipg188.

15. Moro E, Gross RE, Krauss JK. What's new in surgical treatment for dystonia? Mov Disord. 2013;28:1013-20.

16. Bronte-Stewart $\mathrm{H}$, Taira $\mathrm{T}$, Valldeoriola $\mathrm{F}$, et al. Inclusion and exclusion criteria for DBS in dystonia. Mov Disord. 2011; 26(Suppl 1):S5-16.

17. Burke R, Fahn S, Marsden CD, Bressman S, Moskowitz C, Friedman J. Validity and reliability of a rating scale for the primary torsion dystonias. Neurology. 1985;35:73-7.

18. Consky E, Basinski A, Belle L, Ranawaya R, Lang AE. The Toronto Western Spasmodic Torticollis Rating Scale (TWSTRS): assessment of validity and inter-rater reliability. Neurology. 1990;40(suppl 1):445

19. Gavarini S, Vayssière N, Delort $P$, et al. Stereotactic MRI in DYT1 dystonia: focal signal abnormalities in the basal ganglia do not contraindicate deep brain stimulation. Stereotact Funct Neurosurg. 2008;86:245-52.

20. Jahanshahi M, Czernecki V, Zurowski AM. Neuropsychological, neuropsychiatric, and quality of life issues in DBS for dystonia. Mov Disord. 2011;26(Suppl 1):S63-78.

21. Vidailhet M, Vercueil L, Houeto JL, et al. Bilateral deep-brain stimulation of the globus pallidus in primary generalized dystonia. N Engl J Med. 2005;352:459-67.
22. Kupsch A, Benecke R, Müller J, et al. Pallidal deep-brain stimulation in primary generalized or segmental dystonia. N Engl J Med. 2006;355:1978-90

23. Volkmann J, Wolters A, Kupsch A, et al. Pallidal deep brain stimulation in patients with primary generalised or segmental dystonia: 5-year follow-up of a randomised trial. Lancet Neurol. 2012;11:1029-38.

24. Witt JL, Moro E, Ash RS, et al. Predictive factors of outcome in primary cervical dystonia following pallidal deep brain stimulation. Mov Disord. 2013;28:1451-5.

25. Brüggemann N, Kühn A, Schneider SA, et al. Short- and long-term outcome of chronic pallidal neurostimulation in monogenic isolated dystonia. Neurology. 2015;84:895-903.

26. Volkmann J, Mueller J, Deuschl G, et al. Pallidal neurostimulation in patients with medication-refractory cervical dystonia: a randomised, sham-controlled trial. Lancet Neurol. 2014;13:875-84.

27. Kiss ZH, Doig K, Eliasziw M, Ranawaya R, Suchowersky O. The Canadian multicenter trial of pallidal deep brain stimulation for cervical dystonia: preliminary results in three patients. Neurosurg Focus. 2004;17:E5.

28. Pretto TE, Dalvi A, Kang UJ, Penn RD. A prospective blinded evaluation of deep brain stimulation for the treatment of secondary dystonia and primary torticollis syndromes. J Neurosurg. 2008:109:405-9.

29. Skogseid IM, Ramm-Pettersen J, Volkmann J, Kerty E, Dietrichs E, Røste GK. Good long-term efficacy of pallidal stimulation in cervical dystonia: a prospective, observer-blinded study. Eur J Neurol. 2012;19:610-5.

30. Walsh RA, Sidiropoulos C, Lozano AM, et al. Bilateral pallidal stimulation in cervical dystonia: blinded evidence of benefit beyond 5 years. Brain. 2013;136:761-9.

31. Damier P, Thobois S, Witjas T, et al. Bilateral deep brain stimulation of the globus pallidus to treat tardive dyskinesia. Arch Gen Psychiatry. 2007;64:170-6.

32. Mentzel CL, Tenback DE, Tijssen MA, Visser-Vandewalle VE, van Harten PN. Efficacy and safety of deep brain stimulation in patients with medication-induced tardive dyskinesia and/or dystonia: a systematic review. J Clin Psychiatry. 2012;73:1434-8.

33. Vidailhet $M$, Yelnik $J$, Lagrange $C$, et al. Bilateral pallidal deep brain stimulation for the treatment of patients with dystoniachoreoathetosis cerebral palsy: a prospective pilot study. Lancet Neurol. 2009;8:709-17.

34. Koy A, Hellmich M, Pauls KA, et al. Effects of deep brain stimulation in dyskinetic cerebral palsy: a meta-analysis. Mov Disord. 2013;28:647-54.

35. Eltahawy HA, Saint-Cyr J, Giladi N, Lang AE, Lozano AM. Primary dystonia I more responsive than secondary dystonia to pallidal interventions: outcome after pallidotomy or pallidal deep brain stimulation. Neurosurgery. 2004;54:613-9.

36. Deuschl G, Bain P, Brin M. Consensus statement of the Movement Disorder Society on Tremor. Ad Hoc Scientific Committee. Mov Disord. 1998;13(Suppl 3):2-23.

37. Deuschl G, Raethjen J, Hellriegel H, Elble R. Treatment of patients with essential tremor. Lancet Neurol. 2011;10:148-61.

38. Benabid AL, Pollak P, Louveau A, Henry S, de Rougemont J. Combined (thalamotomy and stimulation) stereotactic surgery of the VIM thalamic nucleus for bilateral Parkinson disease. Appl Neurophysiol. 1987;50:344-6.

39. Fasano A, Herzog J, Deuschl G. Selecting appropriate tremor patients for DBS. In: Bain P, Aziz T, Liu X, Nandi D, eds. Deep brain stimulation. Oxford, UK: Oxford University Press; 2009.

40. Deuschl G, Bain P. Deep brain stimulation for tremor [correction of trauma]: patient selection and evaluation. Mov Disord. 2002; 17(Suppl 3):S102-11.

41. Fasano A, Daniele A, Albanese A. Treatment of motor and nonmotor features of Parkinson's disease with deep brain stimulation. Lancet Neurol. 2012;11:429-42.

42. Fasano A, Bove F, Lang AE. The treatment of dystonic tremor: a systematic review. J Neurol Neurosurg Psychiatry. 2014;85:759-69.

43. Espinoza Martinez JA, Arango GJ, Fonoff ET, et al. Deep brain stimulation of the globus pallidus internus or ventralis intermedius nucleus of thalamus for Holmestremor. Neurosurg Rev. 2015;38:753-63. 
44. Alusi SH, Worthington J, Glickman S, Findley LJ, Bain PG. Evaluation of three different ways of assessing tremor in multiple sclerosis. J Neurol Neurosurg Psychiatry. 2000;68:756-60.

45. Wishart HA, Roberts DW, Roth RM, et al. Chronic deep brain stimulation for the treatment of tremor in multiple sclerosis: review and case reports. J Neurol Neurosurg Psychiatry. 2003;74:1392-7.

46. Liu X, Aziz TZ, Miall RC, et al. Frequency analysis of involuntary movements during wrist tracking: a way to identify ms patients with tremor who benefit from thalamotomy. Stereotact Funct Neurosurg. 2000;74:53-62.

47. Mehanna R, Itin I. Which approach is better: bilateral versus unilateral thalamic deep brain stimulation in patients with fragile $\mathrm{X}$-associated tremor ataxia syndrome. Cerebellum. 2014;13:222-5.

48. Leehey MA, Munhoz RP, Lang AE, et al. The fragile X premutation presenting as essential tremor. Arch Neurol. 2003;60:117-21.

49. Bayreuther C, Delmont E, Borg M, Fontaine D. Deep brain stimulation of the ventral intermediate thalamic nucleus for severe tremor in anti-MAG neuropathy. Mov Disord. 2009;24:2157-8.

50. Breit S, Wachter T, Schols L, et al. Effective thalamic deep brain stimulation for neuropathic tremor in a patient with severe demyelinating neuropathy. J Neurol, Neurosurg Psychiatry. 2009;80(2):235-6.

51. Espay AJ, Duker AP, Chen R, et al. Deep brain stimulation of the ventral intermediate nucleus of the thalamus in medically refractory orthostatic tremor: preliminary observations. Mov Disord. 2008;23:2357-62.
52. Lyons MK, Behbahani M, Boucher OK, Caviness JN, Evidente VG. Orthostatic tremor responds to bilateral thalamic deep brain stimulation. Tremor Other Hyperkinet Mov (N Y). 2012;2.

53. Wang D, Sanchez J, Foote KD, et al. Failed DBS for palliation of visual problems in a case of oculopalatal tremor. Parkinsonism Related Disord. 2009;15:71-3.

54. Zesiewicz TA, Shaw JD, Allison KG, Staffetti JS, Okun MS, Sullivan KL. Update on treatment of essential tremor. Current Treat Options Neurol. 2013;15:410-23.

55. Bonuccelli U, Ceravolo R, Salvetti S, et al. Clozapine in Parkinson's disease tremor. Effects of acute and chronic administration. Neurology. 1997;49:1587-90.

56. Shih LC, LaFaver K, Lim C, Papavassiliou E, Tarsy D. Loss of benefit in VIM thalamic deep brain stimulation (DBS) for essential tremor (ET): how prevalent is it? Parkinsonism Related Disord. 2013;19:676-9.

57. Favilla CG, Ullman D, Wagle Shukla A, Foote KD, Jacobson CEt, Okun MS. Worsening essential tremor following deep brain stimulation: disease progression versus tolerance. Brain. 2012;135:1455-62.

58. Herzog J, Hamel W, Wenzelburger R, et al. Kinematic analysis of thalamic versus subthalamic neurostimulation in postural and intention tremor. Brain. 2007;130:1608-25.

59. Young RF, Li F, Vermeulen S, Meier R. Gamma Knife thalamotomy for treatment of essential tremor: long-term results. J Neurosurg. 2010;112:1311-7.

60. Lipsman N, Schwartz ML, Huang Y, et al. MR-guided focused ultrasound thalamotomy for essential tremor: a proof-ofconcept study. Lancet Neurol. 2013;12:462-8. 